\title{
Visualization of Network Vulnerability During Extreme Weather Events for Situation Awareness Enhancement
}

DOI:

10.1109/PESGM.2018.8586494

\section{Document Version}

Accepted author manuscript

Link to publication record in Manchester Research Explorer

\section{Citation for published version (APA):}

Liu, H., Zhou, Y., \& Panteli, M. (2018). Visualization of Network Vulnerability During Extreme Weather Events for Situation Awareness Enhancement. In 2018 IEEE Power and Energy General Meeting

https://doi.org/10.1109/PESGM.2018.8586494

\section{Published in:}

2018 IEEE Power and Energy General Meeting

\section{Citing this paper}

Please note that where the full-text provided on Manchester Research Explorer is the Author Accepted Manuscript or Proof version this may differ from the final Published version. If citing, it is advised that you check and use the publisher's definitive version.

\section{General rights}

Copyright and moral rights for the publications made accessible in the Research Explorer are retained by the authors and/or other copyright owners and it is a condition of accessing publications that users recognise and abide by the legal requirements associated with these rights.

\section{Takedown policy}

If you believe that this document breaches copyright please refer to the University of Manchester's Takedown Procedures [http://man.ac.uk/04Y6Bo] or contact uml.scholarlycommunications@manchester.ac.uk providing relevant details, so we can investigate your claim.

\section{OPEN ACCESS}




\title{
Visualization of Network Vulnerability During Extreme Weather Events for Situation Awareness Enhancement
}

\author{
Haiyang Liu, Yutian Zhou, Member IEEE, and Mathaios Panteli, Member IEEE \\ School of Electrical and Electronic Engineering \\ The University of Manchester, UK \\ haiyang.liu@student.manchester.ac.uk,yutian.zhou@manchester.ac.uk, mathaios.panteli@manchester.ac.uk
}

\begin{abstract}
Extreme events, driven mainly by natural disasters and extreme weather, have severe impacts on the resilient operation of power systems. Adequate situation awareness during these events is essential to the implementation of those preventive and corrective actions that would improve network resilience. It is thus becoming increasingly important to quantify and visualize the network impacts of such extreme events, which would enhance the situation awareness of system operators. Within this context, this paper proposes a fragility-based probabilistic approach to firstly assess the impact of extreme weather events on network integrity and then generate heat maps for visualizing the spatiotemporal vulnerability of the network to the weather event. The application of the proposed approach using a simplified version of the Great Britain transmission network demonstrates that such a tool can benefit the situation awareness of the system operators, enabling their effective and timely response to such disastrous events.
\end{abstract}

Index Terms-Fragility curves, heat maps, natural disaster, power system resilience, situation awareness, weather

\section{INTRODUCTION}

Extreme weather events such as windstorms, snowstorms and floods, may cause significant damage to power systems, resulting in severe power disruptions. Recent experiences are now signifying the increasing importance for power systems to achieve high levels of resilience to such disastrous events $[1,2]$. This is becoming of growing concern given the direct impact of climate change on the frequency and severity of extreme weather events around the world [3].

Among the different strategies that can be applied for boosting power systems resilience to extreme weather events and natural disasters $[1,3]$ (e.g. upgrading the structures with stronger, more robust materials or building redundant transmission routes), advanced visualization and situation awareness [4] is considered critical as it enables the effective and timely decision-making by the system operators, which plays a key role in preserving resilience during emergencies. For instance, user-friendly visualization technologies, including color contour, animated arrows, and dynamically sized pie-charts, could enable transmission and distribution system operators to perform effective system monitoring and develop adequate cognition of the evolving conditions during extreme events. This would in turn support their effective response with the implementation of the most appropriate preventive and corrective actions to improve the resilience performance of the network.

In this light, existing studies [5-7] have adopted visualization technologies based on Geographical Information System (GIS). More specifically, the outage management of distribution networks can be enhanced by visualizing potential damages on overhead lines that are caused by weather related events [5]. Further, the impact of severe weather events on customer damage cost has been quantified and visualized in [6] using a GIS-based technology. This can provide warnings to network operators to implement proper actions in a timely and effective manner. A more recent study in [7] introduced the concept of risk maps, which is essentially the visualization of risk levels of a system under different weather conditions. In fact, the renowned GIS software ArcGIS has developed a specific toolbox (i.e. Storm Vulnerability Assessment) for assessing the impact of extreme weather events on distribution networks [8]. It has to be noted that though visualization technologies are increasingly used in distribution networks, the application to transmission networks is less advanced.

Differently from existing works, this paper proposes a fragility-based probabilistic approach for quantifying and visualizing the evolving vulnerability of an electricity network exposed to extreme weather events. The key contribution of this work is the development of a clear and straightforward method to visualize the structural integrity of and stress on an electricity network during extreme weather events, as well as the integration of probabilistic fragility-curve based modelling into short-term operational decision-making process. In order to determine the weather-dependent failure probability of electricity network components, the concept of fragility curves is used which can effectively relate the failure probability of a component to the weather conditions that it experiences. This is then used to determine the vulnerable components that should be taken into account when generating and visualizing the heat maps of the network vulnerability. Differently from existing works (e.g. [5]-[7]) where the focus was on regional/local analysis at distribution network level, the proposed methodology is applied using a reduced 29-bus representation of the Great Britain (GB) transmission network for visualizing the impact of severe windstorms at a national 
level considering also the spatiotemporal features of such events. This application shows that such a tool can be very beneficial in supporting the system operator's situation awareness and short-term operational decision-making on the most suitable corrective or preventive actions to apply for mitigating the impacts of such extreme events.

This paper is structured as follows: the probabilistic approach for determining the vulnerable components and visualizing the network vulnerability is presented in Section II. In Section III, the proposed approach is illustrated using the reduced 29-bus representation of the GB transmission network. Finally, Section IV concludes the paper.

\section{VISUALIZING THE NETWORK VULNERABILITY DURING EXTREME WEATHER EVENTS}

This section describes the main steps of the proposed approach for quantifying and visualizing the impact of extreme weather events on power systems.

\section{A. Fragility modelling}

A fragility function describes the failure probability of a structure as a function of the intensity of the hazard that it is exposed to (e.g. earthquake, windstorms, etc.), and as such it is a very useful approach for assessing the vulnerability of a network of structures to a hazard.

Fragility curves can be derived through different methods, including: (i) empirically, (ii) experimentally, (iii) analytically, (iv) using expert judgment or (v) through a combination of these methods [9]. Due to the low frequency of extreme events, empirical fragility curves or curves based on expert judgment are difficult to develop due to insufficient experiences and thus statistical data; hence methods (i) and (iv) are ruled out. In this work, analytical fragility curves have been produced, which is a key output of the "Resilient Electricity Networks for Great Britain" (RESNET) project [9]. A generic fragility curve is shown in Fig. 1, whose shape varies and depends on the specific application. This curve can be expressed as follows:

$$
P\left(w_{i}\right)= \begin{cases}0, & \text { if } w<w_{\text {critical }} \\ P(w), & \text { if } w_{\text {critical }} \leq w<w_{\text {collapse }} \\ 1, & \text { if } w \geq w_{\text {collapse }}\end{cases}
$$

where $P\left(w_{i}\right)$ is the failure probability of the component as a function of the weather parameter $w_{i}$ at simulation step $i$, $w_{\text {critical }}$ is the value of the weather parameter at which the weather-dependent failure probability picks-up and $w_{\text {collapse }}$ represents the value of the weather parameter at which the failure of the component is certain, i.e. $P\left(w_{i}\right)=1$.

It can thus be clearly seen that fragility curves are a very effective way for determining the weather-dependent failure probability of each network component. By mapping the profile of the underlying weather parameter on each network component at each and every simulation step, the time- and weather-dependent failure probability of the component can be effectively quantified and used to determine the spatial and temporal vulnerability of the overall network to the extreme event. It has to be clarified that these curves do not relate to the loading conditions of a network component, which are expected to be less impactful than severe weather conditions.

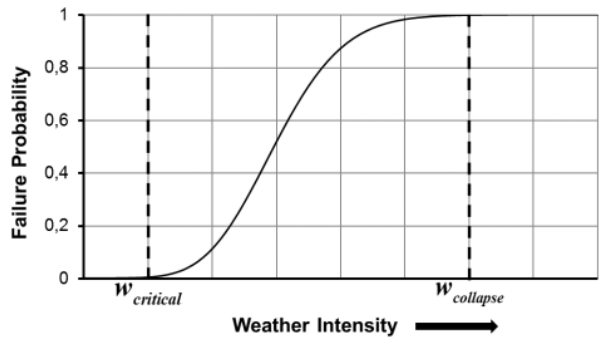

Fig. 1. Generic fragility curve showing the failure probability of a component as a function of the weather intensity

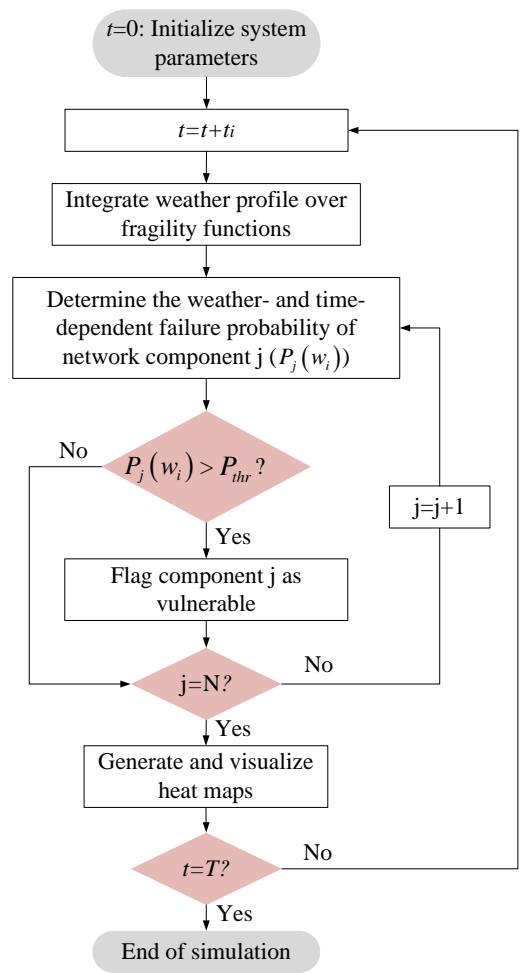

Fig. 2. Time-series procedure for identifying the vulnerable components and generating the heat maps

\section{B. Time-series procedure for generating the spatiotemporal heat maps to visualize network vulnerability}

Fig. 2 shows the time-series procedure for identifying the vulnerable network components and generating the heat maps. The aim of this procedure is to identify the vulnerable network components based on their weather-dependent failure probability, as this is provided by their fragility functions, which will be further used for generating and visualizing the spatiotemporal vulnerability heat maps.

After initializing the system parameters, at simulation step $i$ the weather parameter $w_{i}$ is mapped to the fragility functions to obtain the weather- and time-dependent failure probability of component $j\left(P_{j}\left(w_{i}\right)\right)$ using (1). This $P_{j}\left(w_{i}\right)$ is then compared with a failure probability threshold $\left(P_{t h r}\right)$ to determine if the specific component is highly vulnerable (i.e. prone to fail due to the weather event). There are two main approaches for defining $P_{t h r}$ : (i) it can be set by the system operator based on the desired network resilience (i.e. security level) during severe weather events (e.g. it can be set low to capture as many vulnerable components as possible or it can 
be set high to capture only those components that are greatly affected by the weather event), and (ii) it can be decided using the failure probability distribution of the components exposed to the weather event. Further, $P_{t h r}$ can reflect the system operator's preference on operational robustness. For instance, the lower the $P_{t h r}$ the more lines are vulnerable, the more constrained the operation, but the more the operational robustness (similar to the security criteria of " $n-1$ " or " $n-2$ "). In this work, the probability-based approach is used as it can provide a systematic, probabilistic approach of capturing the impact of the weather event on the vulnerability of each component, and hence of the overall network. If $P_{j}\left(w_{i}\right)>P_{t h r}$, then component $j$ is flagged as vulnerable at simulation step $i$; otherwise, it is recorded as not vulnerable and the simulation procedure proceeds to evaluating the weather-dependent vulnerability of the other components at simulation step $i$.

Once the vulnerability of each component ( $N=$ total number of components) is evaluated for the simulation period $T$, the spatiotemporal heat maps can be generated for visualizing the vulnerability of the network. Different tools can be used for this purpose, such as ArcGIS [10]. It has to be noted that this approach can produce these heat maps very quickly. It can therefore be used to provide a projection of the network vulnerability in the face of an extreme weather event (e.g. hours or days ahead depending on the available projected weather conditions), or it can provide a close to real-time indication of the network vulnerability as the event unfolds. It thus enables the application of both preventive and corrective for mitigating the impacts of the extreme event.

\section{CASE Study Application}

This section presents the application of the proposed approach using a simplified version of the GB transmission network with the aim of developing vulnerability heat maps when the test system is exposed to severe windstorms.

\section{A. Test network and simulation input data}

Fig. 3 shows the 29-bus simplified version of the GB transmission network. This network model consists of 29 nodes, 98 overhead transmission lines in double-circuit configuration and one single circuit transmission line (i.e. between nodes 2 and 3). Further details on this test network can be found in $[9,11]$. Without loss of generality, the test network has been arbitrarily divided to 6 weather regions (which are assumed to have homogenous weather regions) to model the spatial and regional impact of the wind event. Data permitting more regions could be used. In this way, the wind event can travel at any direction across the network.

In order to model a real movement of a wind event across the GB transmission network, the Storm ANGUS in 2016 [12] is considered as a reference to build the windstorm movement profile. Fig. 4 shows Storm ANGUS and its direction used in this work. A simulation time of $24 \mathrm{hrs}$ is considered, which is split to 6 time intervals with duration of 4 hours each. It has to be noted here that any time resolution can be used by the proposed approach, given that the relevant data (e.g. weather data) is available. The direction of the windstorm depicted in

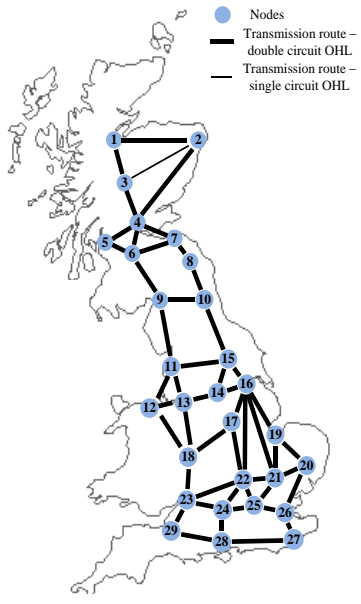

a) Transmission network

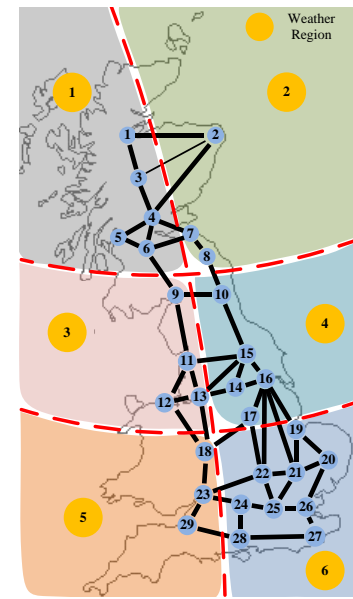

b) Weather regions
Fig. 3. The reduced 29-bus GB transmission network
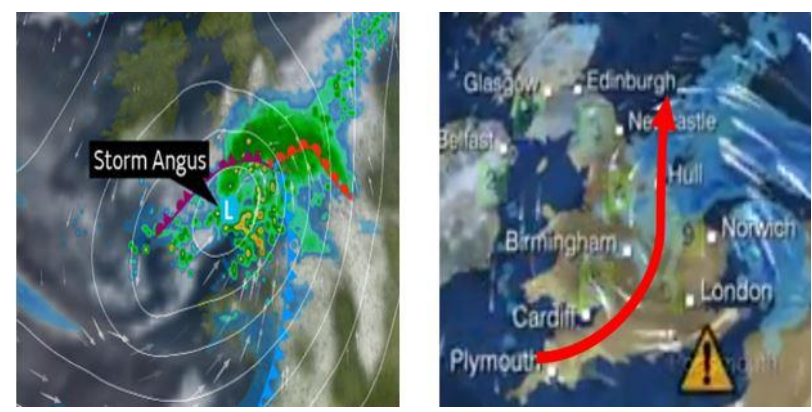

Fig. 4. Satellite cloud image of Storm Angus and its movement [12]

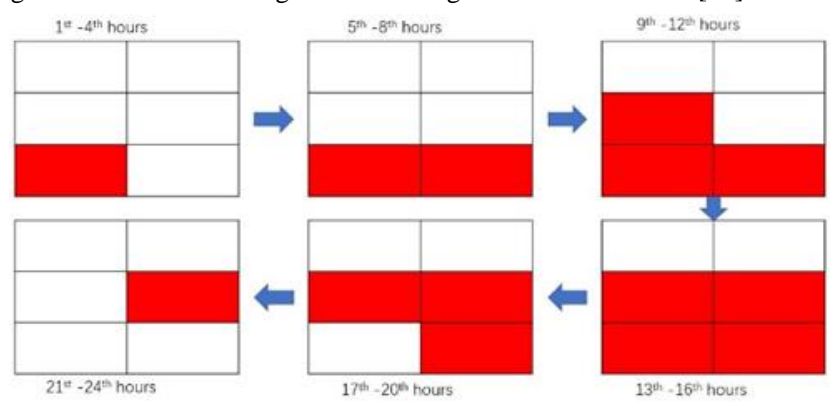

Fig. 5. Affected regions for each time interval

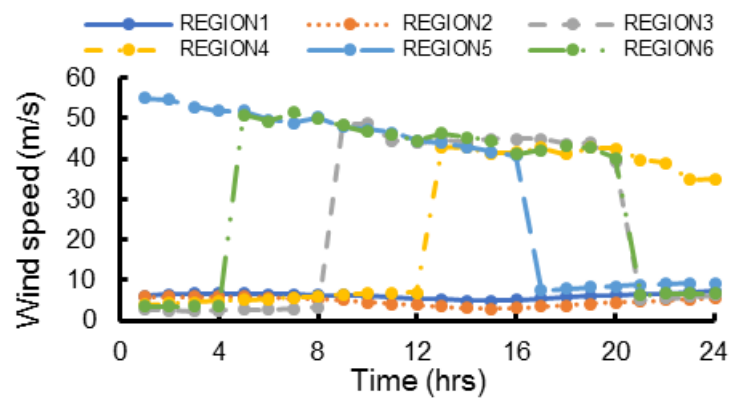

Fig. 6. Time-series wind profiles of the 6 weather regions

Fig. 4 has been transformed to regional direction to determine the affected regions (of the ones in Fig. 3.b) by the windstorm. Fig. 5 presents the affected regions for each time interval used in the simulations. Based on this, Fig. 6 shows the regional windstorm profiles. A peak wind speed of $55 \mathrm{~m} / \mathrm{s}$ is assumed for the $1^{\text {st }}$ hour of the windstorm, which is considered to be 


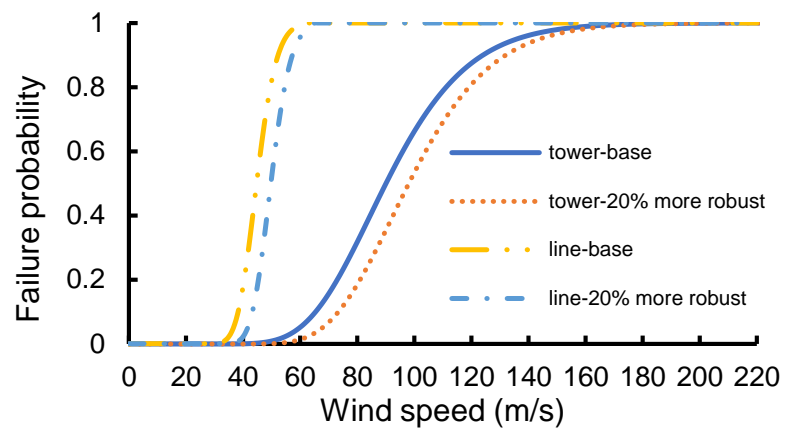

Fig. 7. Windstorm fragility curves of transmission lines and tower (base and robust case studies)

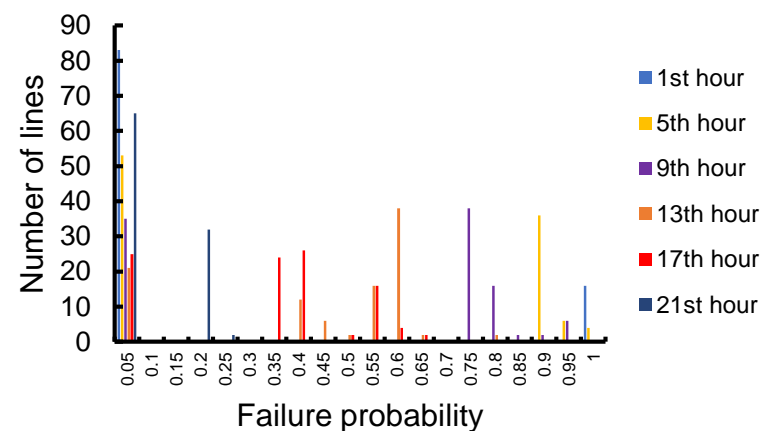

Fig. 8. Failure probability distribution for the $1^{\text {st }}, 5^{\text {th }}, 9^{\text {th }}, 13^{\text {th }}, 17^{\text {th }}$ and $21^{\text {st }}$ hour of the windstorm

reducing as the windstorm moves across the GB transmission network (reaching $35 \mathrm{~m} / \mathrm{s}$ at the end of the windstorm, i.e. the $24^{\text {th }}$ hour). Normal wind conditions are used for the time that a region is not affected by the windstorm. As can be seen in Fig. 5, Regions 1 and 2 are not hit by the windstorm; hence, normal wind conditions are assumed for these regions for the entire simulation period.

The transmission lines and towers are considered as possible vulnerable components to the windstorm. Fig. 7 shows the wind fragility curves of these components. It can be seen that the transmission towers are considered more robust than the transmission lines, since as a structure are stronger to the high wind speeds. The "base" and "robust" case studies are shown in Fig. 7. The robust case study refers to making the components more robust to the windstorm, by arbitrarily shifting the fragility curves by $20 \%$ to the right.

\section{B. Failure probability distribution and threshold $\left(P_{t h r}\right)$}

Fig. 8 shows the failure probability distribution of all the transmission lines for the $1^{\text {st }}, 5^{\text {th }}, 9^{\text {th }}, 13^{\text {th }}, 17^{\text {th }}$ and $21^{\text {st }}$ hour of the windstorm, which hours correspond to the $1^{\text {st }}$ hour of each 4-hour time interval used in the simulations when the peak wind speed for the specific interval is observed for each region (as can be seen in Fig. 6). A step of 5\% was used to divide the probability intervals in the range $[0,1]$. It can be clearly seen that the majority of the line failure probabilities lies within the 0.05 range of failure probability, which is considered to be of low threat to the network resilience. However, higher failure probabilities can pose severe threat to the network vulnerability. Thus, in order to capture and visualize the impact of the windstorm on these lines to the system operator which would enable the implementation of preventive and/or corrective actions, the $P_{t h r}$ is set to be 0.1 . The lines thus with

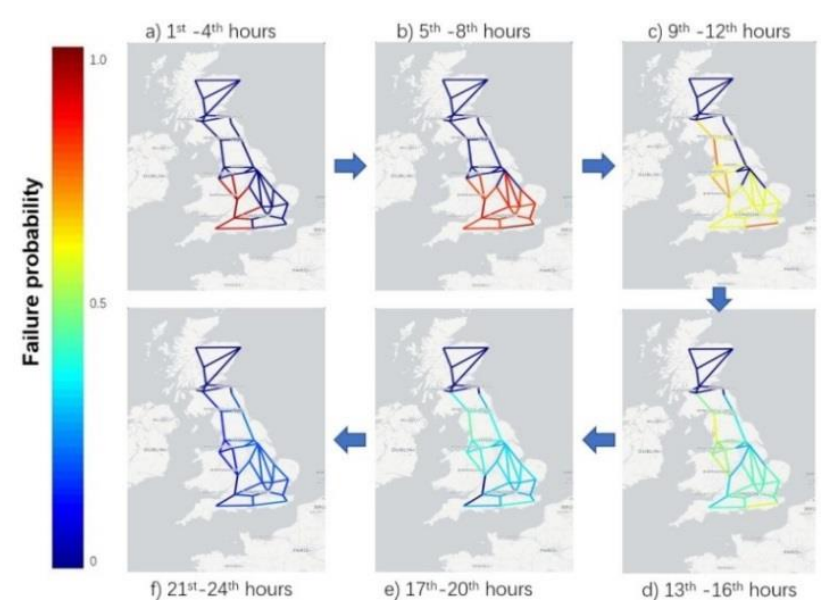

Fig. 9. The transmission network vulnerability heat maps

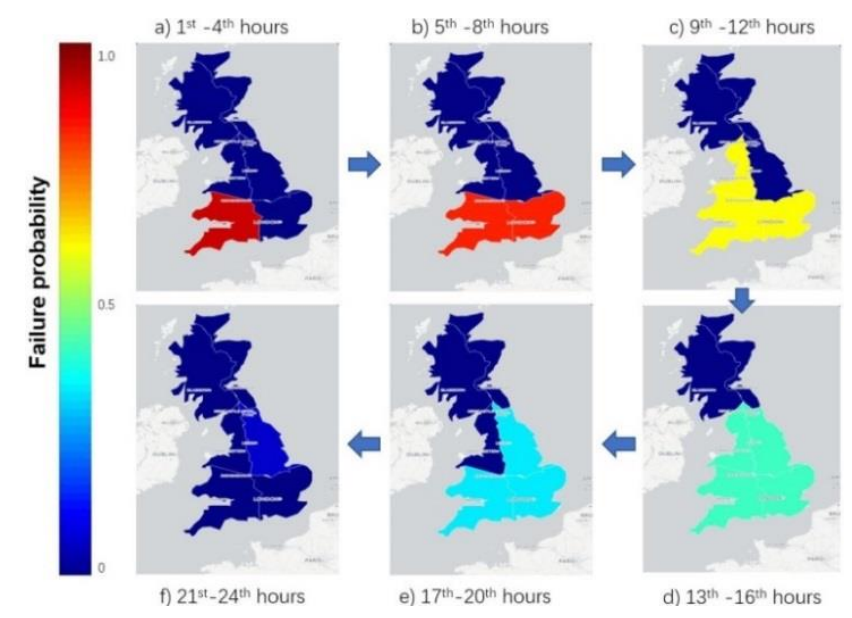

Fig. 10. The regional vulnerability heat maps

a failure probability larger than 0.1 are flagged as vulnerable and considered in the generation of the vulnerability heat maps.

\section{Vulnerability heat maps}

Fig. 9 presents the transmission network vulnerability heat maps for the six 4-hour time intervals of Fig. 5 used in the simulations, while Fig. 10 shows the regional vulnerability maps (corresponding to the six regions of Fig.

3.b). The vulnerability levels are indicated by using successive colors from blue (failure probability equal to zero) to red (failure probability equal to one). This can be adjusted depending on the requirements by the system operator. Also, depending on the data available or on the desired time resolution by the system operator, a different time resolution (e.g. half hourly or hourly) could be used.

It is evident that these illustrations provide a very useful visualization of the network vulnerability (at both component and regional level) of the upcoming (or during) the windstorm. This provides critical insights to the system operators on the areas or components that will be highly vulnerable to the upcoming (or evolving) event, enhancing their awareness of the areas of high risk. This can then effectively support their decision-making on the need to apply those actions to preserve the resilience of the network 


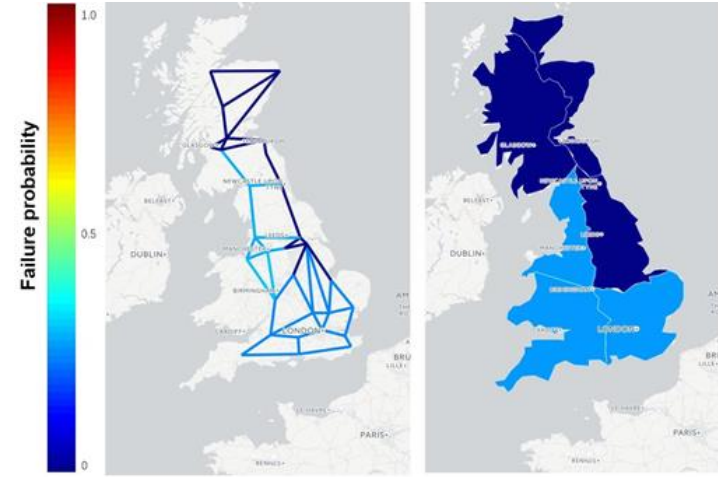

Fig. 11. The transmission network and regional vulnerability heat maps for the "more robust" case study for the 9-12 hours of the windstorm

and mitigate the impacts of the extreme event. For example, based on these maps, the system operator may decide to apply preventive generation re-dispatch to the areas that are of lower risk to the extreme event or proceed to controlled islanding to isolate the areas of high risk and prevent the cascading outages to the rest of the network. In terms of long-term planning, it can also enable the effective planning of resilience investments to boost the robustness of the areas that are of high risk and exposure to such disastrous events.

D. Assessing and visualizing the impact of making the network stronger to the windstorm

Different approaches can be applied for improving the robustness of the transmission network to such extreme weather events. One of these approaches is making the transmission components stronger to the high wind speeds experienced during the windstorm. In order to evaluate the ability of the proposed approach to capture the effect of an infrastructure resilience enhancement strategy, the "robust" case study is next evaluated and the vulnerability heat maps of Figs. 9 and 10 are reproduced. Only the interval of 9-12 hours is presented here (Fig. 11) for demonstration purposes; similar results are obtained for the other intervals. As aforementioned, the "more robust" case study is simulated by shifting the fragility curves of the "base" case study by $20 \%$ to the right (see Fig. 7). It can be clearly seen that the proposed approach can effectively capture the effect of the resilience enhancement strategy of making the network more robust. Compared to Figs. 9.c and 10.c, the visualization of the transmission components' and regional vulnerability changes the color of the exposed components and regions respectively from yellow to blue, meaning that the vulnerability of the exposed components and regions is now lower as a result of increasing the robustness of the transmission lines and towers.

\section{CONCLUSIONS}

A time-series probabilistic procedure has been proposed in this paper to visualize the vulnerability of a power system exposed to extreme weather events. Such a visualization tool is very beneficial in developing and maintaining high levels of situation awareness, which is highly critical for the short-term operational decision making on the most appropriate corrective and preventive measures to preserve the resilience of the system. The concept of fragility curves has been used, which is proven to be a very effective way of relating the failure probability of a component to the weather conditions that it is exposed to.

The illustration of the proposed methodology using the 29-bus simplified version of the GB transmission network demonstrates its capability of capturing and visualizing (through the use of heat maps) the evolving vulnerability of the test system to extreme windstorms. It has to be noted here that the Storm ANGUS was used for demonstration purposes. Any windstorm (including direction and intensity) can be modelled using the proposed approach. The simulation results show that the proposed method can be used not only to support the timely decision-making on the most suitable operational measures for boosting the operational network resilience, but through the illustration of the "robust" case study it has been also shown that such a tool can provide useful insights on the effect of infrastructure resilience enhancement strategies. This can in turn support the planning of impactful and cost-effective resilience investments.

Future work will focus on extending the visualization tool to other extreme weather events, as well as providing a risk indication of the extreme event by generating large number of failure scenarios, combining the probability of a failure scenario with its impact severity. It is also essential to consider the loading conditions of the transmission corridors within a holistic power flow analysis tool and develop tools for implementing appropriate remedial actions.

\section{REFERENCES}

[1] M. Panteli and P. Mancarella, "The Grid: Stronger, Bigger, Smarter?: Presenting a Conceptual Framework of Power System Resilience," IEEE Power \& Energy Magazine, vol. 13, no. 3, pp. 58-66, May 2015.

[2] Y. Wang, C. Chen, J. Wang, and R. Baldick, "Research on Resilience of Power Systems Under Natural Disasters - A Review," IEEE Trans. Power Systems, vol. 31, no. 2, pp. 1604-1613, Mar. 2016.

[3] M. Panteli and P. Mancarella, "Influence of Extreme Weather and Climate Change on the Resilience of Power Systems: Impacts and Possible Mitigation Strategies," Electr. Power Syst. Research, vol. 127, pp. 259-270, 2015.

[4] M. Panteli and D. S. Kirschen, "Situation Awareness in Power Systems: Theory, Concepts and Applications", Electric Power Systems Research, vol. 122, pp. 140-151, May 2015

[5] P. C. Chen, T. Dokic, N. Stokes, D. W. Goldberg and M. Kezunovic, "Predicting weather-associated impacts in outage management utilizing the GIS framework," in 2015 IEEE PES Innovative Smart Grid Technologies Latin America (ISGT LATAM), Montevideo, pp. 417-422.

[6] Q. Yan, T. Dokic and M. Kezunovic, "GIS-based risk assessment for electric power consumers under severe weather conditions," in 2016 18th Mediterranean Electrotechnical Conference (MELECON), Lemesos, Cyprus, pp. 1-6.

[7] Q. Yan, T. Dokic and M. Kezunovic, "Predicting impact of weather caused blackouts on electricity customers based on risk assessment," 2016 IEEE Power and Energy Society General Meeting (PESGM), Boston, MA, pp. 1-5.

[8] Storm Vulnerability Assessment, ArcGIS for Utilities, Esri.[online] http://solutions.arcgis.com/utilities/electric/help/storm-vulnerability/, Last accessed: July 2017

[9] M. Panteli, C. Pickering, S. Wilkinson, R. Dawson, and P. Mancarella, "Power System Resilience to Extreme Weather: Fragility Modelling, Probabilistic Impact Assessment, and Adaptation Measures," IEEE Trans. Power Systems, vol. 32, no. 5, Sept. 2017

[10] ArcGIS, [Online] https://www.arcgis.com/features/index.html, Last accessed: October 2017

[11] M. Belivanis and K. Bell, "Representative GB Network Model: Notes," University of Strathclyde, Glasgow, Scotland, 2011.

[12] Storm Angus, [Online] https://www.metoffice.gov.uk/barometer/ uk-storm-centre/storm-angus, Last accessed: September 2017 